\title{
Looking at emotional words is not the same as reading emotional words: Behavioral and neural correlates
}

\author{
JOSÉ A. HINOJOSA, CONSTANTINO MÉNDEZ-BÉRTOLO, AND MIGUEL A. POZO \\ Instituto Pluridisciplinar, Universidad Complutense de Madrid, Madrid, Spain
}

\begin{abstract}
Recent research suggests that the allocation of attentional resources to emotional content during word processing might be sensitive to task requirements. This question was investigated in two tasks with similar instructions. The stimuli were positive, negative, and neutral nouns. Participants had to identify meaningful words embedded in a stream of non-recognizable stimuli (task 1) or pseudowords (task 2). Task 1 could be successfully performed on the basis of the perceptual features whereas a lexico-semantic analysis was required in task 2. Effects were found only in task 2. Positive nouns were identified faster, with fewer errors and elicited larger amplitude in an early negativity. Also, the amplitude of a late positivity was larger for both positive and negative nouns than for neutral nouns. It is concluded that some degree of linguistic processing is needed to direct attention to the affective content during word processing.
\end{abstract}

Descriptors: Emotion, Language, ERPs

Emotion plays a crucial role in our everyday lives. It has been firmly established that the processing of affective information modulates several cognitive processes, including memory (e.g., Jaeger, Johnson, Corona, \& Rugg, 2009; Tapia, Carretié, Sierra, \& Mercado, 2008) and attention (e.g., Carretié, Hinojosa, Martín-Loeches, Mercado, \& Tapia, 2004; Schupp, Stockburger, Codispoti, Junghofer, Weike, \& Hamm, 2007; Smith, Cacioppo, Larsen, \& Chartrand, 2003). Most of these studies have focused on the processing of affective cues in image presentations. Some authors have claimed that responses to pictorial stimuli are based on a biological predisposition (Öhman, Flykt, \& Esteves, 2001). In contrast, the emotional significance of linguistic stimuli is symbolic, learned, and culture mediated. Even though writing is a powerful tool for generating emotional experiences, research on the impact of affective information during the reading process is scarce. Specifically, it would be interesting to characterize the stages of linguistic processing at which emotional content influences reading and how the level of analysis of linguistic information modulates the allocation of attentional resources to affective processing. The present study focuses on the latter question by recording behavioral measures and event-related potentials (ERPs), which are particularly well-suited to study the temporal characteristics of emotional processes.

Studies on ERPs that have investigated the interaction between language and emotion revealed that the emotional con-

The authors would like to thank the two reviewers for their highly valuable comments on a previous version of this manuscript. This work was supported by grant PSI2009-08607 from the Ministerio de Ciencia e Innovación of Spain

Address reprint requests to: José A. Hinojosa, Instituto Pluridisciplinar, Universidad Complutense de Madrid, 28040 Madrid, Spain. E-mail: hinojosa@pluri.ucm.es notation of words modulates brain activity at several temporal stages and scalp locations. Despite the inconsistent finding of effects as early as the P1-N1 and P2-N2 time windows in some studies (Bernat, Bunce, \& Shevrin, 2001; Scott, O'Donnell, Leuthold, \& Sereno, 2009; Zhang, Lawson, Guo, \& Jiang, 2006; see Kissler, Assadolahi, \& Herbert, 2006 for a review), two effects seem to be particularly related to the processing of the emotional aspects of words. The first of these effects, the 'early posterior negativity' (EPN), peaks around $250 \mathrm{~ms}$ and shows an occipito-temporal distribution (Herbert, Junghofer, \& Kissler, 2008; Kissler, Herbert, Peyk, \& Junghofer, 2007; Kissler, Herbert, Winkler, \& Junghofer, 2009; Schacht \& Sommer, 2009a, $2009 b)$. The EPN effect is an amplitude difference between emotional and neutral stimuli that has been related to effortless initial phases of attention and evaluative processing during access to affective information (Schupp, Junghofer, Weike, \& Hamm, 2004). Whereas most of the studies have revealed that arousal modulates the EPN effects (Herbert et al., 2008; Kissler et al., 2007, 2009), others have reported valence influences, specifically for positive stimuli (Hinojosa, Carretié, Valcárcel, MéndezBértolo, \& Pozo, 2009b; Schupp et al., 2004). Pleasant and unpleasant words are also typically associated with an enhancement of a late positive component (LPC) that peaks around $500 \mathrm{~ms}$ after word onset over centro-parietal brain areas (Carretié, Hinojosa, Albert, López-Martín, de la Gándara et al., 2008; Dillon, Cooper, Grent-'t-Jong, Woldorff, \& LaBar, 2006; Herbert, Kissler, Junghofer, Peyk, \& Rockstroh, 2006; Kanske \& Kotz, 2007; Schapkin, Gusev, \& Kuhl, 2000). The amplitude of this component is especially sensitive to arousal manipulations of the stimuli (Conroy \& Polich, 2007; Hinojosa, Carretié, MéndezBértolo, Míguez, \& Pozo, 2009a; Olofsson, Nordin, Sequeira, \& Polich, 2008). The LPC has been thought to reflect the functional mobilization of attentional resources and the activation of the 
motivational circuits in the brain that reflect emotional engagement (Bradley \& Lang, 2007; Schupp et al., 2007). A clear functional distinction between these effects has been recently established (Codispoti, Ferrari, \& Bradley, 2007; Schupp et al., 2007). Codispoti and collaborators used a picture repetition paradigm to present emotional and neutral images in three habituation blocks and one dishabituation block. These authors found that, whereas the amplitude of the EPN effect decreased across blocks of stimuli in the habituation phase, it recovered after a brief pause between blocks. Also, this decrease across blocks was not modulated by arousal. In contrast, the amplitude of the LPC decreased across blocks without recovering. Unlike the EPN, emotional images continued to elicit larger amplitudes than neutral pictures in every block. In agreement with previous findings, it was concluded that obligatory perceptual processing that is facilitated by active short-term memory accounted for EPN effects, whereas the LPC reflected increased resource allocation due to the motivational relevance of affective cues.

The EPN and the LPC have been reported in a variety of tasks imposing different demands, such as passive viewing (Herbert et al., 2008), lexical decision (Carretié et al., 2008; Kanske \& Kotz, 2007; Schacht \& Sommer, 2009a), or counting a particular grammatical category of words (Kissler et al., 2009). This evidence suggests an automatic allocation of attentional resources to the processing of the emotional content of the words. In contrast, the results of some studies that failed to report either EPN or LPC responses for emotional words (Hinojosa et al., 2009b; Schacht \& Sommer, 2009a) indicate some task-dependence during the processing of affective information. Such a divergent pattern of results raises the question of the level of linguistic processing that is necessary to direct attention to affective content. This question has been the focus of several ERP investigations that have used a different set of tasks imposing distinct demands on language processing. Some of these studies have been limited to the late stages of the processing. Naumann, Bartussek, Diedrich, and Laufer (1992) compared the processing of positive, negative, and neutral adjectives in an affective categorization task and a structural task, in which participants had to decide whether the word was shorter, equal, or longer than six letters. They found an equal increment in the amplitude of the LPC for positive and negative words as compared to neutral words in both tasks, although with a different topography. The authors concluded that there is a qualitative difference between structural and explicit emotional processing. A similar conclusion was reached in a subsequent experiment (Naumann, Maier, Diedrich, Becker, \& Bartussek, 1997), where negative and neutral nouns were presented in a letter-search, a concrete-abstract decision, and an affective categorization task. Only the latter task was associated with larger LPC amplitudes for negative as compared to positive words. Finally, Fischler and Bradley (2006) found emotional modulations of the LPC when participants' attention was directed to affective content but not orthographic or lexical aspects. Therefore, it seems that the processing of affective information reflected by the modulation of the amplitude of the LPC is sensitive to different task demands.

A different set of studies focused on both early and late stages of the processing. In a study by Kissler and co-workers (2009), positive, negative, and neutral words were presented to participants in a silent reading task and a grammatical judgment in which they had to count either nouns or adjectives. Positive and negative words as compared to neutral words elicited enhanced EPN amplitudes in both the passive viewing and the grammatical task. Also, the LPC was larger for pleasant words as compared to negative and neutral words in the silent reading task and for the attended word class. Since there were no interferences between task and emotional content, the authors concluded that the implicit processing of the emotional content of words is independent of a grammatical decision. In Experiment 2 of Schacht and Sommer's study (2009a), the authors investigated how the presence of a minimal previous neutral semantic context influenced affective processing under different task demands by presenting emotional target verbs that were preceded by nouns. Specifically, participants had to perform a lexical decision task, a semantic judgment about whether the target verb was a meaningful follower of the preceding noun, and a structural decision task in which they had to decide whether all the letters within the string of the target verb were written in the same font or not. Schacht and Sommer reported enhanced EPN amplitudes for negative and positive verbs as compared to neutral verbs that were independent of the level of processing. Also, the amplitude of the LPC was larger for positive and negative verbs in all tasks with the exception of the structural task. The authors concluded that the level of the processing of linguistic information influences the access to affective information at late but not at early stages. Interestingly, Experiment 1 investigated emotional processing during single word processing by presenting the same target verbs in a lexical decision task. However, they found a rather different pattern of results consisting of an enhanced EPN for positive and negative verbs with an unusually delayed latency (starting around $320 \mathrm{~ms}$ ) compared to other studies and an absence of LPC modulations. Overall, the results of the study by Schacht and Sommer suggest that the presence of a semantic context interacts with task demands during the processing of affective information.

It is thus noteworthy that previous studies that have explicitly focused on the influence of task demands in the processing of linguistic affective information have either used tasks that did not require lexico-semantic processing explicitly (Kissler et al., 2009), focused on the late stages of the processing (Fischler \& Bradley, 2006; Naumann et al., 1992; Naumann et al., 1997) or provided a semantic context that could have resulted in a facilitated processing of the emotional aspects (Schacht \& Sommer, 2009a). The results of these studies suggest that the emotional effects reflected by the EPN are task-independent. However, the findings of a recent study (Hinojosa et al., 2009b) are problematic for this view. In this study, positive, negative, and neutral nouns as well as non-recognizable stimuli were presented at a high rate of stimulation and with no inter-stimulus interval. Participants had to press a button every time they detected a meaningful word. Interestingly, even though a clear EPN effect was apparent at parieto-occipital regions, there were no amplitude differences between emotional and neutral words. The authors concluded that this lack of effects could be tentatively attributed to the salient perceptual differences between meaningful and non-recognizable stimuli. Under these circumstances, the processing of the linguistic features (including lexico-semantic aspects) of the words was not mandatory in order to successfully perform the task. As a consequence, it seems that emotional content was not processed. To what extent these findings generalize to a situation that forces the processing of lexico-semantic aspects is a question that has not yet been addressed.

The aim of the present study is to further investigate the processing requirements that are necessary to direct attention to affective information at an early and a late stage of single-word 
processing (as indexed by the EPN effect and the LPC, respectively). For this purpose we measured ERPs and behavioral data (Reaction Times [RTs], and errors/omissions) in two indirect tasks with similar instructions but different processing demands. To replicate previous findings, participants were instructed to detect meaningful words embedded in a stream on non-recognizable stimuli in task 1 . No amplitude differences between emotional and neutral words in the time window of the EPN effects and the LPC are expected. In task 2, attention will be directed to the processing of lexico-semantic properties of the words by instructing subjects to identify meaningful words intermingled in a sequence of pseudowords, which constitutes a variant of the typical lexical decision task. In such a task, lexico-semantic information has to be processed for a successful discrimination of words from pseudowords (Fujimaki, Hayakawa, Ihara, Wei, Munetsuna et al., 2009; Ihssen, Heim, \& Keil, 2007; Ray, 2008). Whether differences between emotional and neutral words will be found in the time window of the EPN effect and/or the LPC is not known. Lexico-semantic effects in the same time-range of the EPN and with a similar distribution have been reported (Hauk, Davis, Ford, Pulvermüller, \& Marslen-Wilson, 2006; Hinojosa, Martín-Loeches, Casado, Muñoz, Carretié et al., 2001; MartínLoeches, Hinojosa, Gómez-Jarabo, \& Rubia, 2001; Rudell \& Hua, 1997; see Martín-Loeches, 2007 for a review). Also, some studies found modulations of the amplitude of either the EPN (Scott et al., 2009) or the LPC (Carretié et al., 2008; Scott et al., 2009) elicited by emotional words in typical lexical decision tasks. This divergent pattern of results, which might be due in part to the use of different task parameters and/or stimulus features, shows that research with lexical decision tasks has not led to unequivocal findings. Therefore, although some kind of modulation of the EPN and/or the LPC by directing attention to lexico-semantic features might be hypothesized, strong predictions cannot be made.

\section{Methods}

\section{Subjects}

Thirty-two Spanish native speakers ( 28 female) participated in the study as volunteers. Their mean age was 23 (ranging between 20 and 33 years). They all were right-handed according to the Edinburgh Handedness Inventory (Oldfield, 1971). All participants reported normal or corrected-to-normal visual acuity.

\section{Stimulus Materials and Procedure}

The complete stimulus set consisted of 240 Spanish nouns ( 80 positive, 80 negative, and 80 neutral). Additionally, 240 background stimuli were used in task 1 , and 240 pronounceable and orthographically legal pseudowords in task 2 . Background stimuli were made by cutting the 240 words in several portions. These portions were combined randomly, resulting in non-sense stimuli resembling the physical attributes of the words (size, color, brightness, etc.). All stimuli were presented black-on-white on a computer monitor, controlled by the Gentask module of the STIM2 package (NeuroScan Inc., Charlotte, NC).

Nouns were selected from a previous pilot study. A 720-noun list, divided in three sets ( 240 words each) was evaluated by 45 (15 for each set) subjects, who rated valence, arousal, and the level of concreteness of each noun in a 9-point Likert scale ( 9 being very positive, very arousing, and very concrete, respectively). Division of the 720 words into three sets was made due to the long time that evaluating all words would take for a single sample of subjects. Nouns that were presented to participants in the ERP experiment were selected according to several criteria that were contrasted with one-way analyses of variance (ANOVAs) and post-hoc analyses with the Bonferroni correction (alpha $<0.05$ ). Positive and negative words were matched in arousal but differed in valence. Neutral nouns differed from positive and negative nouns in both valence and arousal. All nouns had similar frequency of use in Spanish (Alameda \& Cuetos, 1995; 65 positive words, 61 negative words, 62 neutral words; $F(2,158)=0.04$; $p>.05)$ and were equated in word length (3 syllable positive nouns, 3 negative nouns, 2.9 neutral nouns; $F(2,158)=1.2$; $p>.05)$. Finally, all were matched on the concreteness scale (6 positive words, 6.4 negative words, 6.3 neutral words; $F(2,158)=1.8 ; p>.05)$. Table 1 summarizes mean ratings for each word emotional category in the valence and arousal dimensions, as well as the results of the ANOVAs.

Every set of positive, negative, and neutral stimuli was divided into two blocks of 40 nouns. Each of these two blocks had similar levels of valence, arousal, concreteness, frequency, and length. The blocks were alternated in tasks 1 and 2 across participants, so every noun was presented once during an experimental session. Figure 1 exemplifies the procedure. Stimuli were arranged in 8 sequences for each of the tasks and presented according to the rapid stream stimulation procedure (Hinojosa, Martín-Loeches, Casado, Muñoz, Fernández-Frías, \& Pozo, 2001; Rudell, 1992; Zhang, Liu, \& Zhang, 2009). This procedure speeds up stimuli processing by presenting them at a high rate (with a stimulus onset asynchrony [SOA] of $300 \mathrm{~ms}$ ). In each of these sequences the computer displayed mainly background stimuli (task 1) or pseudowords (task 2). After four to seven of these stimuli (this number being randomized), a test stimulus was presented. Each sequence included 15 test stimuli (5 positive, 5 negative, and 5 neutral nouns), together with the proportional amount of background stimuli (task 1) or pseudowords (task 2). The order of the test stimuli was pseudo-randomly determined, with the constraint of no more than two of the same type occurring consecutively.

Participants performed two indirect tasks. In task 1, they had to discriminate meaningful words embedded in a sequence of background stimuli by pressing a button as fast as possible every time a word was detected. Our previous study (Hinojosa et al., $2009 \mathrm{~b}$ ) showed that it is not necessary to process the linguistic properties of the nouns in order to discriminate words from nonsense stimuli. The lack of amplitude differences in those waves

Table 1. Means of Valence (1, negative to 9, positive) and Arousal (1, calming to 9, arousing) Assessments Given by the Independent Samples of Subjects to Each Word Type

\begin{tabular}{lcc}
\hline \hline WORDS & Valence & Arousal \\
\hline Positive & 7.8 & 6.7 \\
Negative & 2.3 & 6.8 \\
Neutral & 5 & 5 \\
One-way ANOVA & $F(2,158)=4606.7$ & $F(2,158)=214.5$ \\
(Affect Type) on each factor & $p<.0001$ & $p<.0001$ \\
\hline \hline
\end{tabular}

Note: Last row shows the results of the statistical analyses for each of these variables. Post-hoc Bonferroni analyses are reported in the main text. 

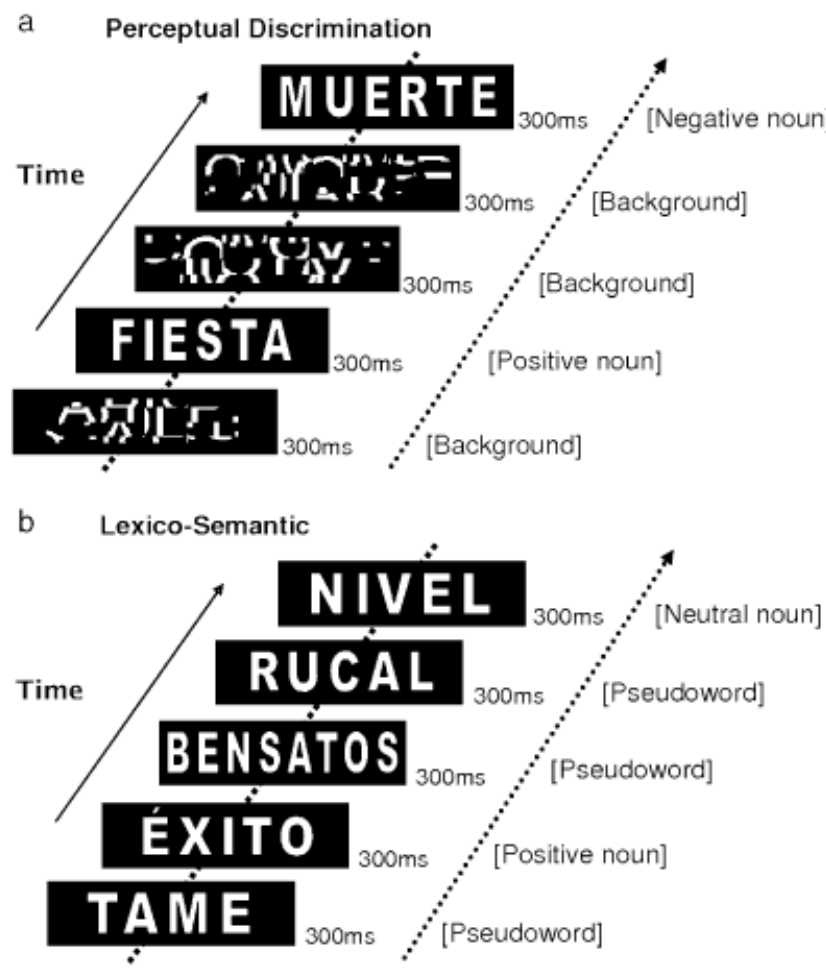

Figure 1. Schematic illustration of the stimulation paradigm for both tasks: A. Perceptual discrimination task $($ Muerte $=$ Death; Fiest $a=$ Party; B. Lexico-semantic task (Nivel $=$ Level; Éxito $=$ Success $)$.

associated to affective processing suggested that target stimuli might be identified on the basis of their great perceptual discrepancy with background stimuli. Participants received the same instruction in task 2, that is, to identify meaningful words. However, nouns were presented in a stream of pseudowords instead of background stimuli. In this case, an analysis of the lexico-semantic features of the words is necessary in order to discriminate them from pseudowords, since both stimuli are perceptually similar. Even though this is a highly demanding task (brief stimulus presentation with no stimulus interval), it has proven to be useful for research in semantics (Pu, Peng, Demaree, Song, Wei, $\& \mathrm{Xu}, 2005)$.

All 8 sequences of each task were presented consecutively. Each participant received one of eight orders designed to minimize stimulus order effects. In half of the eight presentation orders, task 1 was presented first. The remaining four presentation orders began with task 2 . A practice sequence was presented prior to the first sequence of each task. Participants were told to minimize blinking during stimulus presentation.

\section{Data Acquisition}

Electroencephalographic data were recorded using an electrode cap (Electro-Cap International, Eaton, $\mathrm{OH}$ ) with tin electrodes. A total of 62 electrodes homogenously distributed over the entire scalp were used (see Figure 2). All were referenced to the linked mastoids. Bipolar horizontal and vertical electrooculogram was recorded for artifact rejection purposes. Electrode impedances were kept below $3 \mathrm{~K} \Omega$. The signals were recorded continuously with a bandpass from 0.1 to $50 \mathrm{~Hz}(3 \mathrm{~dB}$ points for $-6 \mathrm{~dB}$ octave roll-off) and a digitization sampling rate of $250 \mathrm{~Hz}$.

\section{Data Analysis}

Trials with RTs longer than $1500 \mathrm{~ms}$ or shorter than $200 \mathrm{~ms}$ were not analyzed. Also, those trials with omissions and false alarms were excluded from the analyses. Data were filtered from 0.1 to $30 \mathrm{~Hz}$. Eye movements were corrected using the method described by Semlitsch, Anderer, Schuster, and Preelich (1986). Remaining artifacts were removed after visual inspection. Data was segmented from $300 \mathrm{~ms}$ before to $800 \mathrm{~ms}$ after word onset and baseline corrected using the entire $300 \mathrm{~ms}$ before word onset as baseline. When stimulus order is counterbalanced across different stimulus categories as in this study, content-related differences in baseline activity are cancelled out, and no differences in baseline activity are to be expected (Herbert et al., 2008; Kissler et al., 2009; Pu et al., 2005). After the averaging of every stimulus category, data were re-referenced to an average reference.

Overall repeated-measures ANOVAs were first conducted to compare amplitudes between the ERPs elicited by positive, negative, and neutral nouns in the two tasks. Separate analyses were performed for the EPN and LPC effects. Amplitude was measured as the mean voltage within a particular time interval. To avoid a loss of statistical power when repeated-measures ANOVAs are used to quantify large numbers of electrodes (Oken \& Chiappa, 1986), eleven regions of interest were computed out of the 62 electrodes. The activity across electrodes was averaged within each region of interest (ROI). These regions are shown in Figure 2.

The ANOVA included three within-subjects factors: Task (two levels: lexico-semantic and perceptual discrimination), Affective Type (three levels: positive, negative, and neutral), and Region of Interest (11 levels). The Greenhouse-Geisser epsilon correction was applied to adjust the degrees of freedom of the $F$-ratios where necessary. In a second step and in order to explore possible interactions involving topographical factors, further ANOVAs were conducted for each particular ROI with Affective Type and Task as within-subjects factors. $P$-values were adjusted to the Bonferroni correction for multiple comparisons $(p<.05)$. Finally, follow-up planned comparisons with the Bonferroni correction $(p<.05)$ were made for determining the significance of pairwise contrasts where appropriate.

\section{Results}

\section{Behavioral Data}

There were 3,840 epochs in each task (40 averages per each of the 3 word categories in 32 participants). In the perceptual discrimination task, the mean number of errors was $0.6(s . d .=0.9)$ for positive nouns, 0.5 (s.d. $=0.8)$ for negative nouns and 0.7 $(s . d .=0.8)$ for neutral nouns. In the lexico-semantic task, the mean number of errors was $6.7($ s.d. $=3.5)$ for positive nouns, $10.4(s . d .=4.2)$ for negative nouns and $12.3(s . d .=4.5)$ for neutral nouns. The interaction between Task and Affective Type was significant $(F(2,62)=32.2 ; p<.0001)$. Further post-hoc analyses with the Bonferroni correction $($ alpha $<0.05)$ showed that positive words elicited fewer omissions than negative and neutral words in the lexico-semantic task. No other comparison reached significance.

Regarding RTs, in the perceptual discrimination task, mean RTs were $328.6 \quad($ s.d. $=30.6)$ for positive words, 329.7 $(s . d .=34.3)$ for negative words and $329.6($ s.d. $=31.8)$ for neutral words. In the lexico-semantic task, mean RTs were 543.5 


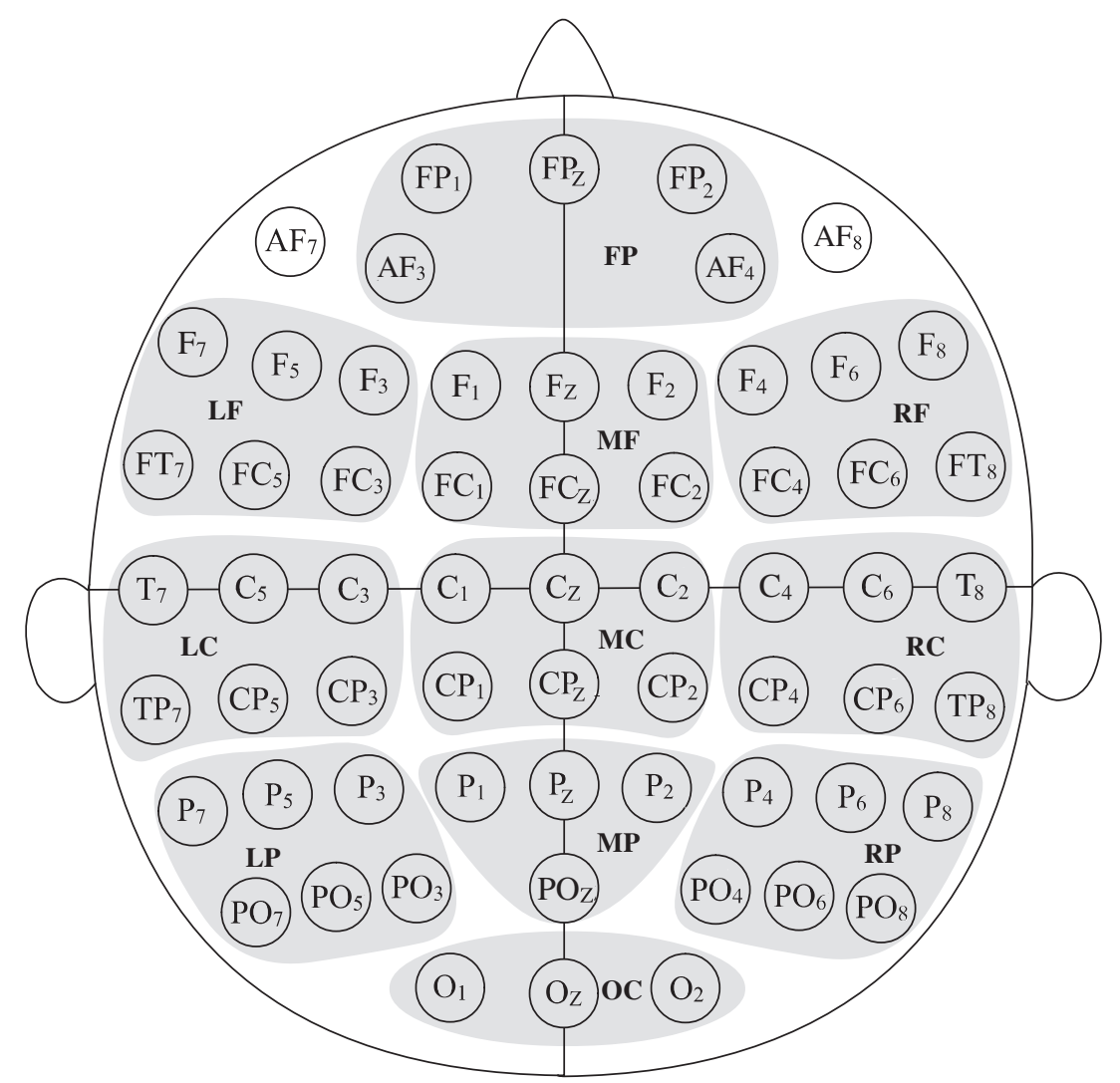

Figure 2. Scalp regions in which ERPs were grouped for statistical contrasts. $F P=$ Frontopolar, $L F=$ Left Frontal; $M F=$ Middle Central; $R F=$ Right Central; $L C=$ Left Central; $M C=$ Middle Central; $R C=$ Right Central; $L P=$ Left Parietal; $C P=$ Central Parietal; $R P=$ Right Parietal; $O C=$ Occipital.

(s.d. $=47.2)$ for positive words, $574($ s.d. $=48.5)$ for negative words, and $580.5(s . d .=55.4)$ for neutral words. The ANOVA revealed significant effects for the Task and Affective Type interaction $(F(2,62)=14.6 ; p<.0001)$. Post-hoc analyses showed that positive words were associated with shorter RTs as compared to negative and neutral words in the lexico-semantic task. Table 2 shows mean and standard deviation of RTs and omissions for every word type in both tasks.

Behavioral data suggest that the perceptual discrimination task was easier to perform than the lexico-semantic task, as reflected by shorter RTs and fewer errors. This conclusion is also supported by fewer false alarms $(F(1,31)=31.1 ; p<.0001)$ in the former $($ mean $=4.3$, s.d. $=2.4)$ as compared to the latter task $($ mean $=14.8$, s.d. $=10.1)$

Table 2. Means and Standard Deviations (in parenthesis) of Behavioral Data for Positive, Negative, and Neutral Words

\begin{tabular}{lrrrrrr}
\hline \hline \multicolumn{3}{c}{ Positive } & \multicolumn{2}{c}{ Negative } & \multicolumn{2}{c}{ Neutral } \\
\hline Perceptual Discrimination & & & & \\
RT (ms) & 328 & $(30.6)$ & 329 & $(34.3)$ & 329 & $(31.8)$ \\
Omissions & 0.6 & $(0.9)$ & 0.5 & $(0.8)$ & 0.7 & $(0.8)$ \\
Lexico-Semantic & & & & & & \\
RT (ms) & 543 & $(47.2)$ & 574 & $(48.5)$ & 580 & $(55.4)$ \\
Omissions & 6.7 & $(4.2)$ & 10.4 & $(3.5)$ & 12.3 & $(4.5)$ \\
\hline \hline
\end{tabular}

\section{Electrophysiological Data}

A selection of the grand averages for positive, negative, and neutral nouns in the two tasks is shown in Figure 3. Two main effects were noticeable. ${ }^{1}$ The first of these effects was a posterior negative component with a frontal positive counterpart, peaking around $250 \mathrm{~ms}$ after stimulus presentation (EPN), with differences notes between emotional and neutral nouns in the lexicosemantic task. The second effect was a central positive component with an onset around $500 \mathrm{~ms}$ (LPC). For statistical purposes, the amplitude of the EPN was measured in the $225-300 \mathrm{~ms}$ time-interval and the amplitude of the LPC in the $550-650 \mathrm{~ms}$ time-interval. Figure 4 shows the topographic difference maps of the distribution of the EPN and the LPC for every emotional category in the two tasks after subtracting the activity associated to neutral words. As indicated in the introduction, our aim was to study task effects during the processing of emotional content, so

\footnotetext{
${ }^{1}$ Several components were also noticeable at parieto-occipital electrodes before the EPN effects in both tasks. These positivities reflect a driving rhythm at the rate of image presentation (approximately every $300 \mathrm{~ms}$ ) that has been repeatedly observed with the use of the rapid stream stimulation paradigm (see Rudell, 1992, and Martín-Loeches, 2007, for an extensive discussion on this issue). Since, at least from a linguistic point of view, pseudowords are more complex stimuli than backgrounds, additional resources might be involved in their processing. This would account for the larger amplitudes for pseudowords that are observed in the lexico-semantic task as compared to those associated to background processing in the perceptual discrimination task.
} 
A
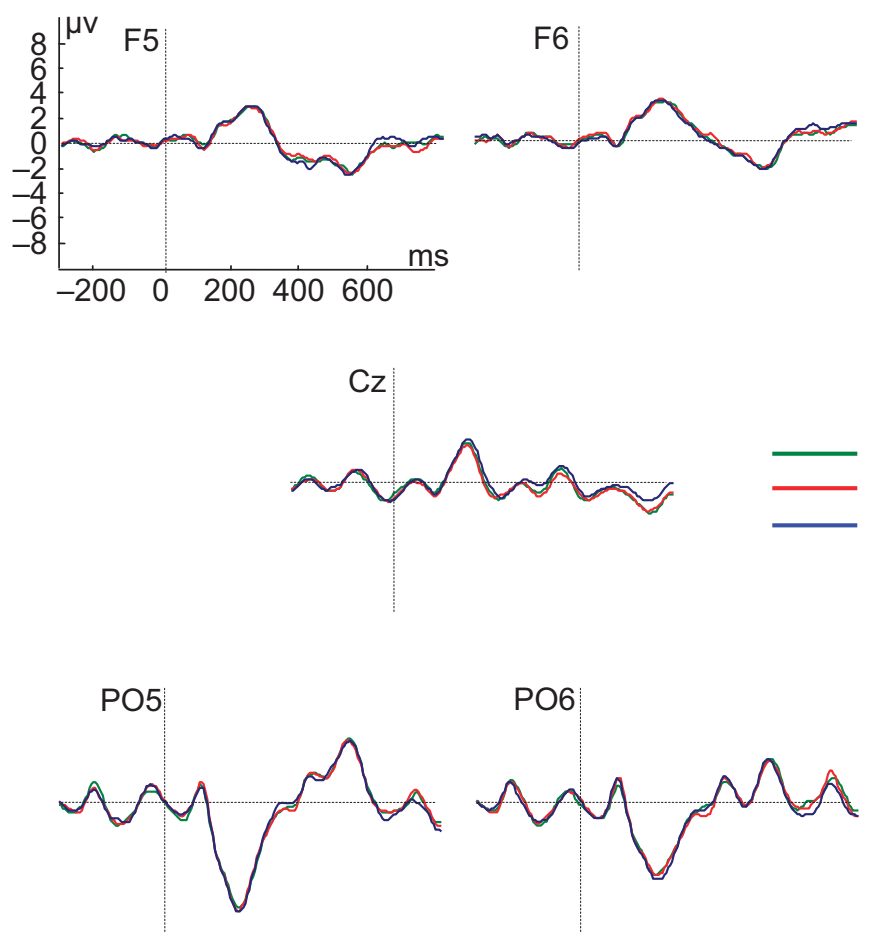

B

\section{Lexico-Semantic}
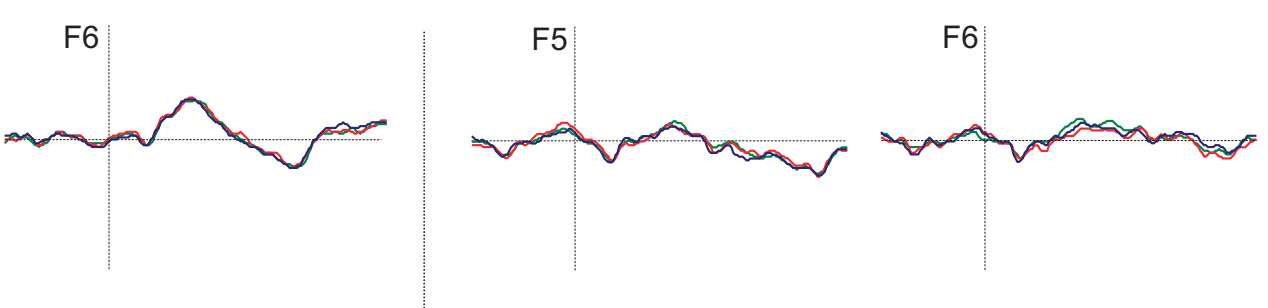

Figure 3. Grand averaged ERPs elicited by negative, neutral, positive nouns at a selected sample of electrodes in (A). Perceptual discrimination task and (B). Lexico-semantic task. Scales are represented at the F5 electrode.

for the sake of clarity only the interactions involving Affective Type and Task are further considered.

225-300 effects (EPN). The results of the overall ANOVA revealed a significant effect of the interaction Task and Affective Type $(F(2,62)=3.6 ; p<.05)$. The interaction between Task and Affective Type and ROI $(F(20,620)=3.3 ; p<.05)$ was also significant. The interaction between Task and Affective Type reached significance at occipital $(F(2,62)=4 ; p<.05)$, right parietal $(F(2,62)=10.6 ; p<.0001)$, middle parietal $(F(2,62)=4$; $p<.05)$ and middle frontal $(F(2,62)=4.4 ; p<.05)$ regions. A statistical trend was found at left frontal $(F(2,62)=3 ; p=.06)$ and right frontal $(F(2,62)=3 ; p=.06)$ regions. In the lexicosemantic task, differences between positive words and both neutral and negative words were noticeable at several ROIs. Positive nouns elicited larger amplitudes than negative and

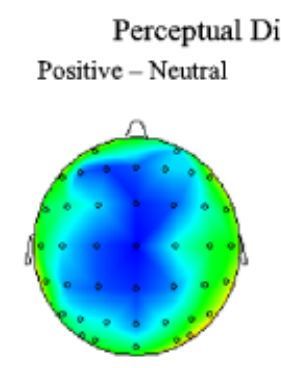

$$
\text { Negative-Neutral }
$$
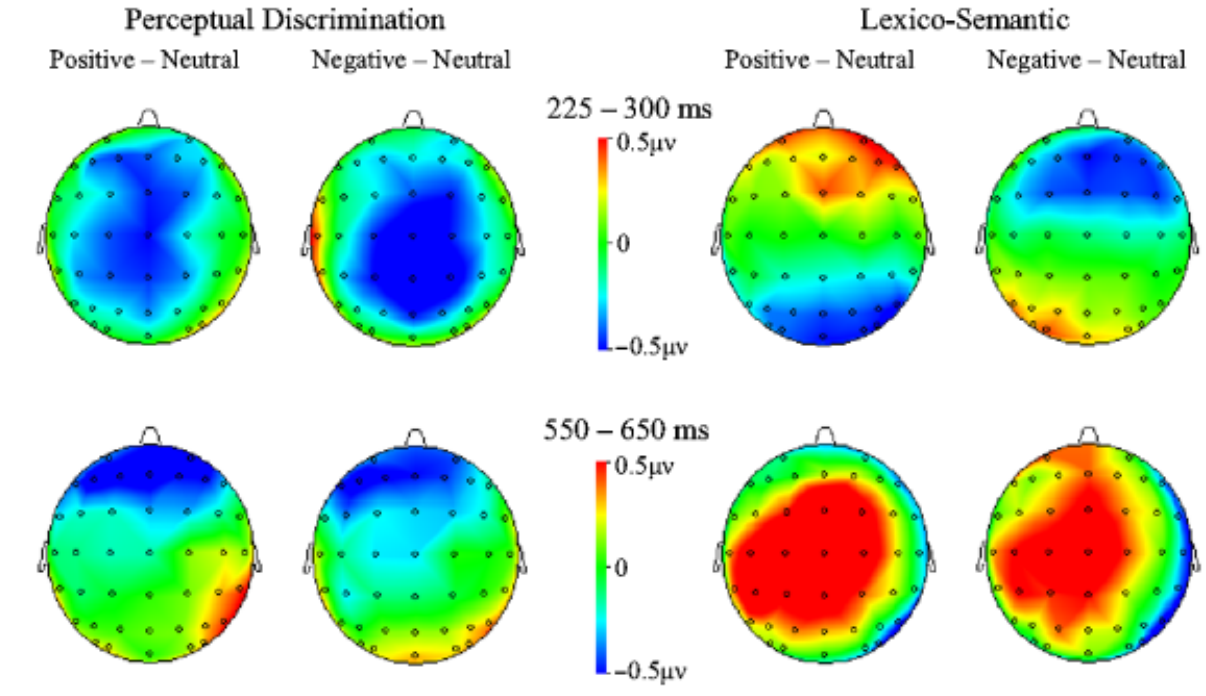

Figure 4. Topographic difference maps of the distribution of the EPN and the LPC for every word emotional category in (A). Perceptual discrimination task and (B). Lexico-semantic task. The activity associated to neutral nouns has been subtracted from the activity elicited by emotional nouns. 
neutral nouns at right $(p<.005$ for the comparison between positive and negative nouns and $p<.0001$ for the comparison between positive and neutral words) and middle parietal regions $(p<.05$ for the positive vs. negative words comparison and $p<.005$ for the positive vs. neutral nouns comparison). Positive words were associated with enhanced amplitudes as compared to negative words in occipital $(p<.05$ and middle frontal regions $(p<.05)$. It should be noted that there were no significant differences at any ROI between negative and neutral words (with $p$ values ranging from .478 to 1 ). No differences were evident in the perceptual discrimination task in this time window. ${ }^{2}$

550-650 effects (LPC). The interaction between Task and Affective Type and ROI reached significance in the overall ANOVA $(F(20,620)=2.4 ; p<.05)$. The Task by Affective Type interaction was significant at occipital $(F(2,62)=5.5 ; p<.05)$, right parietal $(F(2,62)=6.9 ; \quad p<.005), \quad$ middle central $(F(2,62)=4.1 ; p<.05)$ and right central $(F(2,62)=4.3 ; p<.05)$ regions, but only in the lexico-semantic task. Post-hoc analyses showed that positive and negative nouns elicited larger amplitudes than neutral nouns at all these ROIs (with $p$ values ranging from .002 at right parietal to .047 at right central electrodes in the comparison between positive and neutral words and ranging from .022 at occipital to .038 at right parietal electrodes in the negative vs. neutral nouns comparison). There were no significant differences between positive and negative words at any ROI (with $p$ values ranging from .556 to .999).

\section{Discussion}

The major finding of the current study is that the processing of emotional content is affected by the level of analysis of linguistic information during single-word processing. When the participant's attention was directed to the analysis of lexico-semantic features for a successful task performance, positive words were associated with shorter RTs, fewer errors, and larger amplitudes of an early negativity than neutral and negative words. Also, in this task, positive and negative nouns elicited enhanced amplitudes of a late positive component as compared to neutral nouns. In contrast, neither behavioral nor electrophysiological measures were modulated when the task could be done without directing attention to the lexico-semantic properties of the stimuli. Therefore, our data extends previous findings of modulations of several affective-related ERP components by different task demands, including grammatical decisions (Kissler et al., 2009) or affective categorization (Herbert et al., 2006).

It has been proposed that RTs are sensitive to participants' decision-making processes and task-related strategies (Kounios \& Holcomb, 1992; Zhang et al., 2006). The pattern of RTs reported here, as well as the error rate, clearly reveals the existence of such task-related differences in the processing of affective information. The perceptual discrimination task was associated with shorter RTs and fewer errors than the lexico-semantic task. This reflects the fact that participants chose different approaches

\footnotetext{
${ }^{2}$ Both reviewers suggested the possibility that EPN effects emerged at a different latency in the perceptual discrimination task. In order to explore this possibility, we conducted repeated-measures ANOVAs (Affective Type: positive, negative, and neutral) and ROI (11 levels) at every 30$\mathrm{ms}$ time intervals, starting from $100 \mathrm{~ms}$ and extending to $550 \mathrm{~ms}$. None of these analyses reached significance for either the factor Affective Type $(F(2,62)=0.01-1.89 ; p=0.16-0.99)$ or the interaction between Affective Type and ROI $(F(20,620)=0.28-1.30 ; p=0.25-0.97)$.
}

for performing the two tasks even though the instructions were the same for both of them. The absence of differences between emotional and neutral stimuli in behavioral data in the perceptual discrimination task is in agreement with the findings of our previous study (Hinojosa et al., 2009b). This lack of effects could be at least partially attributed to the great perceptual differences between words and background stimuli. This makes the task easy to perform on the basis of this discrepancy so it might not be necessary to process lexico-semantic aspects of words in order to successfully discriminate them from background. As a consequence, attention is not directed to the affective content of the words. In contrast, positive words elicited shorter RTs and fewer errors than negative and neutral words when lexico-semantic aspects needed to be processed in order to achieve a successful task performance. This is in line with previous research that has reported that positive words speed lexical decision times (Carretié et al., 2008; Kanske \& Kotz, 2007; Kuchinke, Jacobs, Grubich, Vo, Conrad, \& Herrmann, 2005; Kuchinke, Vo, Hofmann, \& Jacobs, 2007; Williamson, Harpur, \& Hare, 1991). All in all, behavioral data supports a processing advantage for positive nouns when participants processed the lexico-semantic features of the words.

In agreement with behavioral measures and previous research (Hinojosa et al., 2009b), no EPN effects were found in the perceptual-discrimination task. Again, a failure to process the lexico-semantic properties of the words might explain this lack of effects. In the lexico-semantic task, positive words were associated with larger amplitudes of the EPN, starting at around 200 $\mathrm{ms}$, in posterior regions as compared to negative and neutral words. EPN effects with similar onsets and topographical distributions for emotional words have been reported (Herbert et al., 2008; Kissler et al., 2007; Schacht \& Sommer, 2009a). However, most of these studies found that both positive and negative stimuli equally differed from neutral stimuli, thus reflecting a general arousal effect. Interestingly, in agreement with current data a different set of studies has found that only positive words (Schacht \& Sommer, 2009b), or positive pictures (Hinojosa et al., $2009 \mathrm{~b}$ ) modulated the amplitude of the EPN effect. Although the reason for this discrepancy is still unclear and might be attributed to differences in experimental parameters, these data suggest that some brain circuits might be specifically engaged in the processing of positive effects (see Burgdorf \& Panksepp, 2006 for a comprehensive review of this question). This assertion is also supported by fMRI data (Hamann \& Mao, 2002; Herbert, Ethofer, Anders, Junghofer, Wildgruber et al., 2009; Kensinger \& Schacter, 2006). The finding of a specific effect for positive stimulation might be better accommodated with the proposals made by theories of basic emotions (e.g., Ekman, 1992; Panksepp, 1998; see Posner, Russell, \& Peterson, 2005 for a review), which assume that a discrete and independent neural system subserves every emotion. Nevertheless, to what extent the EPN is specifically sensitive to valence or arousal differences remains a question for future research.

The EPN is thought to reflect visual attention to emotional content that results in a privileged processing of affective information (Schupp, Junghofer, Weike, \& Hamm, 2003). This advantage has been proposed to arise from bidirectional connections between limbic structures and extrastriate areas (Kissler et al., 2006). In support of this view, the activity of the visual extrastriate cortex and the amygdale has been found to correlate during reading of pleasant adjectives (Herbert et al., 2009). Also, the neural generators of a negativity with a similar 
latency and topographical distribution that is sensitive to visuosemantic processing have been located in extrastriate cortices, namely the fusiform/lingual giri (Dien, Frishkoff, Cerbone, \& Tucker, 2003; Hinojosa, Martín-Loeches, Gómez-Jarabo, \& Rubia, 1999; Martín-Loeches et al., 2001). The results of previous research suggested that the processes reflected by the EPN might be automatically triggered and task-independent. In this regard, Kissler and colleagues (2009) found similar EPN modulations by the emotional content of words in both a grammatical decision and a silent reading task. However, in this study the processing of words at different linguistic levels was necessary for a successful performance of the tasks since some authors have argued that passive viewing enhances emotional processing (Schacht \& Sommer, 2009a).

In a further attempt to investigate this question, Schacht and Sommer (2009a) used a structural decision and a lexical decision task that resembled in some aspects those used here. In the structural decision task, participants had to assess whether all the letters of the verbs were written in the same font or not. In the lexical task, they had to decide whether a particular stimulus was a word or a pseudoword. The authors reported that positive and negative words elicited enhanced EPN amplitudes as compared to neutral words in the two tasks and concluded that this effect is unaffected by task requirements. However, the results of the current study are at variance with this view since we did not find EPN differences in a perceptual discrimination task that shared with the structural decision task the rather shallow processing requirements. Despite the existence of some differences between the experimental setting of both studies, the different pattern of results might be related to task requirements in Schacht and Sommer's structural decision task and those in the perceptual discrimination task used in this study. It might be argued that participants had to scan the whole word to compare letter fonts in the former task. This would allow some kind of linguistic analysis to the participants, even at a lexico-semantic level (Schacht and Sommer, 2009a). However, our perceptual discrimination task could be easily performed on the basis of low level visual features such as spatial frequency (low spatial frequencies in words but not in the backgrounds), so it was not necessary to read words and access their lexico-semantic properties for a successful performance of the task. Therefore, our data complement the results of Schacht and Sommer's study by showing that when lexicosemantic features are not processed attention is directed away from affective content.

Concerning the LPC effects, no amplitude differences between emotional and neutral nouns were noticeable in the perceptual discrimination task. This lack of effects has been extensively discussed in a previous study (Hinojosa et al., $2009 b$ ). In contrast, in the lexico-semantic task, both positive and negative words were associated with larger LPC amplitudes that peaked around $550 \mathrm{~ms}$, as compared to neutral words. This effect has been repeatedly reported in the literature (Herbert et al., 2009; Schapkin et al., 2000; Scott et al., 2009). The amplitude of the LPC has been thought to reflect the allocation of attentional resources, stimuli evaluation, and initial memory storage during the processing of emotional content (Cuthbert, Schupp, Bradley, Birbaumer, \& Lang, 2000; Hajcak \& Nieuwenhuis, 2006; Olofsson et al., 2008; Schupp, Cuthbert, Bradley, Cacioppo, Ito, \& Lang, 2000). A number of ERP studies have shown that differences in the amplitude of this component emerged when the processing of several linguistic aspects was necessary for a successful task performance, while they disappeared in tasks that demanded a superficial processing of linguistic properties (Fischler \& Bradley, 2006; Kissler et al., 2009; Naumann et al., 1992; Schacht \& Sommer, 2009a). Despite the existence of some differences in the experimental designs, our data is in agreement with these findings by showing that word emotional content captures the attention at late stages of processing when lexico-semantic aspects are processed but not when this type of information is obviated.

A final comment should be made on the differences between the EPN that was modulated only by positive content and the LPC that was influenced by positive and negative stimuli. In agreement with recent findings (Codispoti et al., 2007; Schupp et al., 2007), our data point to a functional dissociation of those processes indexed by both components, by showing that visual processing and the subsequent allocation of attentional resources might be differentially modulated by affective information during single-word processing.

Overall, the current study shows that the emotional content of words is not always able to attract attentional resources. Affective information captured attention at early and late stages only when lexico-semantic aspects were processed for a successful task performance, so it seems that minimum linguistic analysis is required for emotion effects to emerge. In line with these findings, some authors have pointed out that the extraction of emotional significance of words may require the activation of semantic/conceptual representations (Fischler \& Bradley, 2006; Schacht \& Sommer, 2009b). These results might also accord with the proposal that emotional stimuli need to exceed a critical threshold value before they are able to capture attention (Koster, Crombez, Van Damme, Verschuere, \& De Houwer, 2004; Mogg \& Bradley, 1998). This threshold is influenced by several factors, the degree of involvement of the ongoing cognitive task (Schwartz, Vuilleumier, Hutton, Maravita, Dolan, \& Driver, 2005) and the attentional load (Lavie, 1995, 2005; Vuilleumier, 2005) being two of the most representative. It seems that, when words are to be discriminated from non-word stimuli and such discrimination can be successfully achieved without processing linguistic aspects, their emotional content is not able to surpass the threshold and engage attention.

\section{REFERENCES}

Alameda, J. R., \& Cuetos, F. (1995). Diccionario de frecuencias de las unidades lingüísticas del castellano. Oviedo: Universidad de Oviedo.

Bernat, E., Bunce, S., \& Shevrin, H. (2001). Event-related brain potentials differentiate positive and negative mood adjectives during both supraliminal and subliminal visual processing. International Journal of Psychophysiology, 42, 11-34.

Bradley, M. M., \& Lang, P. J. (2007). Emotion and motivation. In J. T. Cacioppo, L. G. Tassinary, \& G. Berntson (Eds.), Handbook of Psy- chophysiology (2nd ed, pp. 581-607). New York: Cambridge University Press.

Burgdorf, J., \& Panksepp, J. (2006). The neurobiology of positive emotions. Neuroscience and Behavioural Reviews, 30, 173-187.

Carretié, L., Hinojosa, J. A., Albert, J., López-Martín, S., de la Gándara, B. S., Igoa, J. M., \& Sotillo, M. (2008). Modulation of ongoing cognitive processes by emotionally intense words. Psychophysiology, $45,188-196$. 
Carretié, L., Hinojosa, J. A., Martín-Loeches, M., Mercado, F, \& Tapia, M (2004). Automatic attention to emotional stimuli: Neural correlates. Human Brain Mapping, 22, 290-299.

Codispoti, M., Ferrari, V., \& Bradley, M. M. (2007). Repetition and event-related potentials: Distinguishing early and late processes in affective picture perception. Journal of Cognitive Neuroscience, 19, 577-586.

Conroy, M. A., \& Polich, J. (2007). Affective valence and P300 when stimulus arousal level is controlled. Cognition and Emotion, 21, 891901.

Cuthbert, B. N., Schupp, H. T., Bradley, M. M., Birbaumer, N., \& Lang, P. J. (2000). Brain potentials in affective picture processing: Covariation with autonomic arousal and affective report. Biological Psychology, 52, 95-111.

Dien, J., Frishkoff, G. A., Cerbone, A., \& Tucker, D. M. (2003). Parametric analysis of event-related potentials in semantic comprehension: Evidence for parallel brain mechanisms. Cognitive Brain Research, 15, 137-153.

Dillon, D. G., Cooper, J. J., Grent-'t-Jong, T., Woldorff, M. G., \& La Bar, K. S. (2006). Dissociation of event-related potentials indexing arousal and semantic cohesion during emotional word encoding. Brain and Cognition, 62, 43-57.

Ekman, P. (1992). An argument for basic emotions. Cognition and Emotion, 6, 169-200.

Fischler, I., \& Bradley, M. M. (2006). Event-related potentials studies of language and emotion: Words, phrases and task effects. Progress in Brain Research, 156, 185-203.

Fujimaki, N., Hayakawa, T., Ihara, A., Wei, Q., Munetsuna, S., Terazono, Y., et al. (2009). Early neural activation for léxico-semantic access in the left anterior temporal area analyzed by and fMRI-assisted MEG multidipole method. NeuroImage, 44, 1093-1102.

Hajcak, G., \& Nieuwenhuis, S. (2006). Reappraisal modulates the electrocortical response to unpleasant pictures. Cognitive, Affective \& Behavioral Neuroscience, 6, 291-297.

Hamann, S., \& Mao, H. (2002). Positive and negative emotional verbal stimuli elicit activity in the left amygdala. NeuroReport, 13, 15-19.

Hauk, O., Davis, M. H., Ford, M., Pulvermüller, F., \& Marslen-Wilson, W. D. (2006). The time course of visual word recognition as revealed by linear regression analysis of ERP data. NeuroImage, 30, 13831400.

Herbert, C., Ethofer, T., Anders, S., Junghofer, M., Wildgruber, D., Grodd, W., \& Kissler, J. (2009). Amygdala activation during reading of emotional adjectives-An advantage for pleasant content. Social Cognitive and Affective Neuroscience, 4, 35-49.

Herbert, C., Junghofer, M., \& Kissler, J. (2008). Event-related potentials to emotional adjectives during reading. Psychophysiology, 45, 487498.

Herbert, C., Kissler, J., Junghofer, M., Peyk, P., \& Rockstroh, B. (2006). Processing of emotional adjectives: Evidence from startle EMG and ERPs. Psychophysiology, 43, 197-206.

Hinojosa, J. A., Carretié, L., Méndez-Bértolo, C., Míguez, A., \& Pozo, M. A. (2009a). Arousal contributions to affective priming. Emotion, 9, 164-171.

Hinojosa, J. A., Carretié, L., Valcárcel, M. A., Méndez-Bértolo, C., \& Pozo, M. A. (2009b). Electrophysiological differences in the processing of affective information in words and pictures. Cognitive, Affective \& Behavioral Neuroscience, 9, 173-189.

Hinojosa, J. A., Martín-Loeches, M., Casado, P., Muñoz, F., Fernández-Frías, C., \& Pozo, M. A. (2001). Studying semantics in the brain: The rapid stream stimulation paradigm. Brain Research Protocols, 8, 199-207.

Hinojosa, J. A., Martín-Loeches, M., Casado, P., Muñoz, F., Carretié, L., Fernández-Frías, C., \& Pozo, M. A. (2001). Semantic processing of open- and closed-class words: An event-related potentials study. Cognitive Brain Research, 11, 397-407.

Hinojosa, J. A., Martín-Loeches, M., Gómez-Jarabo, G., \& Rubia, F. (1999). Common basal extrastriate areas for the semantic processing of words and pictures. Clinical Neurophysiology, 111, 552-560.

Ihssen, N., Heim, S., \& Keil, A. (2007). The costs of emotional attention: Affective processing inhibits subsequent lexico-semantic analysis. Journal of Cognitive Neuroscience, 19, 1932-1949.

Jaeger, A., Johnson, J. D., Corona, M., \& Rugg, M. D. (2009). ERP correlates of the incidental retrieval of emotional information: Effects of study-test delay. Brain Research, 1269, 105-113.
Kanske, P., \& Kotz, S. A. (2007). Concreteness in emotional words: ERP evidence from a hemifield study. Brain Research, 1148, 138-148.

Kensinger, E. A., \& Schacter, D. L. (2006). Processing emotional pictures and words: Effects of valence and arousal. Cognitive, Affective \& Behavioral Neuroscience, 6, 110-126.

Kissler, J., Assadollahi, R., \& Herbert, C. (2006). Emotional and semantic networks in visual word processing: Insights from ERP studies. Progress in Brain Research, 156, 147-183.

Kissler, J., Herbert, C., Peyk, P., \& Junghofer, M. (2007). Buzzwords: Early cortical responses to emotional words during reading. Psychological Science, 18, 475-480.

Kissler, J., Herbert, C., Winkler, I., \& Junghofer, M. (2009). Emotion and attention in visual word processing. Biological Psychology, 80, $75-83$.

Koster, E. H., Crombez, G., Van Damme, S., Verschuere, B., \& De Houwer, J. (2004). Does imminent threat capture and hold attention? Emotion, 4, 312-317.

Kounios, J., \& Holcomb, P. J. (1992). Structure and process in semantic memory: Evidence from event-related brain potentials and reaction times. Journal of Experimental Psychology: General, 121, 459-479.

Kuchinke, L., Jacobs, A., Grubich, C., Vo, M.L, Conrad, M., \& Herrmann, M. (2005). Incidental effects of emotional valence in single word processing: An fMRI study. NeuroImage, 28, 1022-1032.

Kuchinke, L., Vo, M. L., Hofmann, M., \& Jacobs, A. M. (2007). Pupillary responses during lexical decisions vary with word frequency but not emotional valence. International Journal of Psychophysiology, 65, $132-140$.

Lavie, N. (1995). Perceptual load as a necessary condition for selective attention. Journal of Experimental Psychology: Human Perception and Performance, 21, 451-468.

Lavie, N. (2005). Distracted and confused?: Selective attention under load. Trends in Cognitive Sciences, 9, 75-82.

Martín-Loeches, M. (2007). The gate for reading: Reflections on the recognition potential. Brain Research Reviews, 53, 89-97.

Martín-Loeches, M., Hinojosa, J. A., Gómez-Jarabo, G., \& Rubia, F. J. (2001). An early electrophysiological sign of semantic processing in basal extrastriate areas. Psychophysiology, 38, 114-124.

Mogg, K., \& Bradley, B. P. (1998). A cognitive-motivational analysis of anxiety. Behaviour Research and Therapy, 36, 809-848.

Naumann, E., Bartussek, D., Diedrich, O., \& Laufer, M. E. (1992). Assessing cognitive and affective information processing functions of the brain by means of the late positive complex of the event-related potential. Journal of Psychophysiology, 6, 285-298.

Naumann, E., Maier, S., Diedrich, O., Becker, G., \& Bartussek, D. (1997). Structural, semantic, and emotion-focused processing of neutral and negative nouns: Event-related potential correlates. Journal of Psychophysiology, 11, 234-256.

Öhman, A., Flykt, A., \& Esteves, F. (2001). Emotion drives attention: Detecting the snake in the grass. Journal of Experimental Psychology: General, 130, 466-478.

Oken, B. S., \& Chiappa, K. H. (1986). Statistical issues concerning computerized analysis of brainwave topography. Annals of Neurology, 19, 493-494.

Oldfield, R. C. (1971). The assessment and analysis of handedness: The Edinburgh Inventory. Neuropsychologia, 9, 9-97.

Olofsson, J. K., Nordin, S., Sequeira, H., \& Polich, J. (2008). Affective picture processing: An integrative review of ERP findings. Biological Psychology, 77, 247-265.

Panksepp, J. (1998). Affective neuroscience: The foundations of human and animal emotions. New York: Oxford University Press.

Posner, J., Russell, J. A., \& Peterson, B. S. (2005). The circumplex model of affect: An integrative approach to affective neuroscience, cognitive development, and psychopathology. Development and Psychopathology, 17, 715-734.

Pu, J., Peng, D., Demaree, H. A., Song, Y., Wei, J., \& Xu, L. (2005). The recognition potential: Semantic processing or the detection of differences between stimuli? Cognitive Brain Research, 25, 273-282.

Ray, S. (2008). An investigation of time course of category and semantic priming. Journal of General Psychology, 135, 133-148.

Rudell, A. P. (1992). Rapid stream stimulation and the recognition potential. Electroencephalography and Clinical Neurophysiology, 83, 7782.

Rudell, A. P., \& Hua, J. (1997). The recognition potential, word difficulty, and individual reading ability: On using event-related potentials 
to study perception. Journal of Experimental Psychology: Human Perception and Performance, 4, 1170-1195.

Schacht, A., \& Sommer, W. (2009a). Time course and task dependence of emotion effects in word processing. Cognitive, Affective, \& Behavioral Neuroscience, 9, 28-43.

Schacht, A., \& Sommer, W. (2009b). Emotions in word and face processing: Early and late cortical responses. Brain and Cognition, 69, 538-550.

Schapkin, S. A., Gusev, A. N., \& Kuhl, J. (2000). Categorization of unilaterally presented emotional words: An ERP analysis. Acta Neurobiologiae Experimentalis, 60, 17-28.

Schupp, H. T., Cuthbert, B. N., Bradley, M. M., Cacioppo, J. T., Ito, T., \& Lang, P. J. (2000). Affective picture processing: The late positive potential is modulated by motivational relevance. Psychophysiology, 37, 257-261.

Schupp, H. T., Stockburger, J., Codispoti, M., Junghofer, M., Weike, A. I., \& Hamm, A. O. (2007). Selective visual attention to emotion. Journal of Neuroscience, 27, 1082-1089.

Schupp, H. T., Junghofer, M., Weike, A. I., \& Hamm, A. O. (2003). Emotional facilitation of sensory processing in the visual cortex. Psychological Science, 14, 7-13.

Schupp, H. T., Junghofer, M., Weike, A. I., \& Hamm, A. O. (2004). The selective processing of briefly presented pictures: An ERP analysis. Psychophysiology, 41, 441-449.

Schwartz, S., Vuilleumier, P., Hutton, C., Maravita, A., Dolan, R. J., \& Driver, J. (2005). Attentional load and sensory competition in human vision: Modulation of fMRI responses by load at fixation during taskirrelevant stimulation in the peripheral visual field. Cerebral Cortex, $15,770-786$.
Scott, G. C., O’Donnell, P. J., Leuthold, H., \& Sereno, S. C. (2009). Early emotion word processing: Evidence from event-related potentials. Biological Psychology, 80, 95-104.

Semlitsch, H. V., Anderer, P., Schuster, P., \& Preelich, O. (1986). A solution for reliable and valid reduction of ocular artifacts applied to the P300 ERP. Psychophysiology, 23, 695-703.

Smith, N. K., Cacioppo, J. T., Larsen, J. T., \& Chartrand, T. L. (2003). May I have your attention please: Electrophysiological responses to positive and negative stimuli. Neuropsychologia, 41, 171-183.

Tapia, M., Carretié, L., Sierra, B., \& Mercado, F. (2008). Incidental encoding of emotional pictures: Affective bias studied through event related brain potentials. International Journal of Psychophysiology, 68, 193-200.

Vuilleumier, P. (2005). How brain beware: Neural mechanisms of emotional attention. Trends in Cognitive Sciences, 9, 585-594.

Williamson, S., Harpur, T. J., \& Hare, R. D. (1991). Abnormal processing of affective words by psychopaths. Psychophysiology, 28, 260273.

Zhang, Q., Lawson, A., Guo, C., \& Jiang, Y. (2006). Electrophysiological correlates of visual affective priming. Brain Research Bulletin, 71, 316-323.

Zhang, Y., Liu, Q., \& Zhang, Q. (2009). The recognition potential reflects an intermediate level of visual representation. Neuroscience Letters, 454, 86-90.

(Received May 4, 2009; ACCePted August 3, 2009) 